\section{Nachfolge-Antihistaminikum schafft Rhinitis „mit links“}

\author{
Am Rande der 41. Tagung der Deutschen Dermatologischen \\ Gesellschaft, 1.-5. Mai 2001 in Berlin, gab Prof. Claus Bachert (Gent) \\ ein Interview anlässlich der deutschen Zulassung von Levocetirizin, \\ dem Nachfolgepräparat des Antihistaminikums Cetirizin.
}

\begin{abstract}
Prof. Bachert, Sie waren an ARIA ? (Allergic Rhinitis and its Impact on Asthma) der WHO beteiligt, die den Begriff der "allergischen Rhinitis" neu definiert hat. Welche klinischen Folgen ergeben sich daraus?
\end{abstract}

Bachert: Die WHO ist dazu übergegangen, die Verläufe nicht mehr in ,saisonal" und „perennial" einzuteilen, sondern in ,intermittierend" und „persistierend“. Wenn also mittelschwere Symptome einer allergischen Rhinitis über vier Wochen und mehr als vier Tage pro Woche anhalten, sprechen wir von einer persistierenden Erkrankung.

Diese Definitions-Änderung hat zwei Gründe: Erstens, die Entzündungsreaktion persistiert in aller Regel auch im symptomfreien Intervall, und zweitens ist die Definition des Asthma bronchiale ebenso eingeteilt.

Die neue Differenzierung trifft die Situation unserer Patienten viel besser, weil wir wissen, dass der Patient, der lediglich auf ein einziges Allergen reagiert, sehr selten geworden ist. Selbst ein so genannter "Heuschnupfen-Allergiker" reagiert heute auf Birken-, Gräser- und Kräuterpollen und hat damit eine sehr lang anhaltende Krankheit, die den Begriff „saisonal“ nicht mehr verdient.

\footnotetext{
Sollten alle Patienten mit einer inter- mittierenen oder persistierenden Rhinitis Antihistaminika einnehmen, um die Entwicklung eines allergischen Asthma bronchiale zu verhindern?

Bachert: Der Vorbeugung des allergischen Asthma bronchiale räumen wir vor allem in der Pädiatrie hohe Bedeutung ein, weil wir wissen, dass Kinder mit atopischem Ekzem oder Rhinitis später Asthma bekommen können. Im Erwachsenenalter ist das nicht mehr der vorrangige Behandlungsgrund. Hier stehen die Symptome selbst und die oft
}

stark beeinträchtigte Lebensqualität im Vordergrund. Bei Patienten älter als 30 bis 40 Jahre fürchten wir die Entwicklung eines Asthma weniger.

Auf der anderen Seite gibt es aber viele Patienten, die während der Pollensaison nicht nur Rhinitis-Symptome haben, sondern zusätzlich auch AsthmaBeschwerden. Hier verspreche ich mir natürlich von einer Behandlung mit einem Antihistaminikum, dass ich die erforderliche Asthma-Medikation zum Beispiel mit ß2-Agonisten - reduzieren und auch die Symptomatik besser in den Griff bekommen kann.

Bei persistierender Rhinitis, z.B. bei Hausstaubmilben-Allergie, konnte

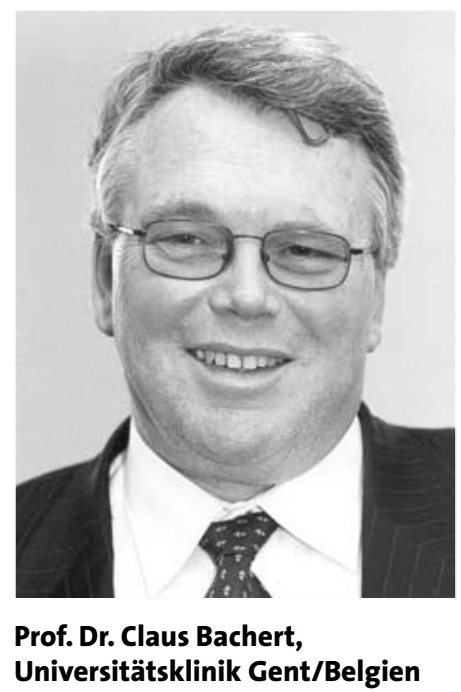
das Antihistaminikum Levocetirizin im Durchschnitt eine 30-35\%ige Symptomreduktion erzielen. Ist das befriedigend? Bachert: Eine 35\%ige Symptomreduktion ist mehr als eine Plazebo-Wirkung und durchaus akzeptabel und gut. Sie liegt vermutlich im oberen Bereich der möglichen Wirksamkeit. Für den $\mathrm{Pa}$ tienten ist das nicht immer befriedigend. Er muss dann weitere Maßnahmen ergreifen, etwa die Allergen-Karenz verstärken oder topische Steroide einsetzen.

Wenn jemand durch ein Antihistami- nikum eine sehr gute Symptomfreiheit erreichen kann, darf er sich dann seinem Allergen aussetzen?

Bachert: Grundsätzlich sagt der Allergologe, dass der Kontakt mit dem
Allergen vermieden werden muss. Praktisch ist das natürlich nicht immer möglich. Unter dem Antihistaminikum kann ich sehr viel besser mit dem Allergen umgehen als ohne dieses Medikament - sowohl in Hinblick auf die vordergründige Symptomatik als auch auf die zu Grunde liegende entzündliche Reaktion.

\section{Prof. Bachert, darf also die Katze wie- - der ins Haus?}

Bachert: Bei Tierhaar-Allergien würde ich nicht dazu raten, sich leichtfertig zu exponieren, denn Tierhaare sind sehr aggressive Allergene. Die Katze sollte also auch nach optimaler Einstellung auf ein zuverlässiges, modernes Antihistaminikum draußen bleiben.

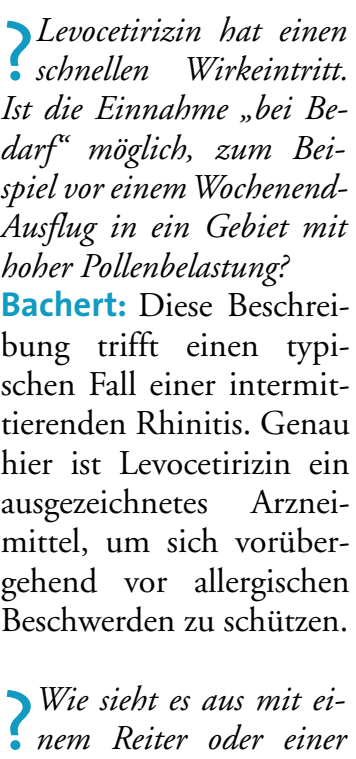
Reiterin mit Pferdehaar-Allergie?

Bachert: Wenn es lediglich darum geht, zweimal im Monat reiten zu gehen, dann kann man sich sicherlich mit Levocetirizin in dieser Zeit gut schützen. Geht man täglich in den Pferdestall, so droht trotz des Schutzes durch das Antihistaminikum bei fortgesetzter Allergen-Exposition die Ausbildung eines allergischen Asthmas - zumindest haben wir bis heute nur sehr indirekte Hinweise darauf, dass ein Antihistaminikum die Neigung, ein Asthma zu entwickeln, auch im Erwachsenenalter verringert.

UCB-Satelliten-Symposium „Ein weiterer Schritt in der Allergie-Therapie“ 